\title{
EXPERIMENTAL STUDY OF THE LASER-INDUCED OXYHEMOGLOBIN PHOTODISSOCIATION IN CUTANEOUS BLOOD VESSELS
}

\author{
A. Gisbrecht ${ }^{1}$ and S. Mamilov ${ }^{2}$ \\ ${ }^{1}$ Institute of Electronics, Bulgarian Academy of Sciences \\ ${ }^{2}$ Institute of Applied Problems of Physics and Biophysics, Academy of Sciences of Ukraine
}

Summary. A new optical method for reduction of local tissue hypoxia is proposed. It is shown that this method of phototherapy allows the control of a local oxygen concentration in tissue. Different aspects of biomedical application of this phenomenon are discussed. The results of in vivo experimental investigation of the laser-induced photodissociation of oxyhemoglobin in cutaneous blood vessels and its role in tissue oxygenation are presented. The rates of oxygen saturation $\mathrm{SpO}_{2}$ in blood and their dependence on the wavelength of the transcutaneous laser irradiation have been experimentally measured.

Key words: oxyhemoglobin, tissue oxygenation, hypoxia, phototherapy, photodissociation

\section{INTRODUCTION}

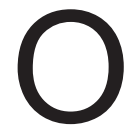
xygen plays a vital role in human cell metabolism; it is a primary mechanism in energy production in tissue. It is well established that hypoxia usually complicates the efficiency of therapeutic methods that strongly depend on the tissue oxygen concentration [1, 2]. Tissue hypoxia may complicate healing of wounds, bedsores, burns, tumors. The deficit of oxygen in tissue is the major problem limiting the efficiency of the phototherapy (including photodynamic therapy) [3, 4].

Therefore, improvement of oxygenation to eliminate tissue hypoxia remains one of the actual problems in modern medicine. Currently it is accepted that adequate tissue concentration of oxygen for normal cell metabolism should exceed 40 $\mathrm{mm} \mathrm{Hg}$. Concentration below $20 \mathrm{~mm} \mathrm{Hg}$ indicates deep hypoxia that leads to tissue necroses. 
In clinical practice the method of lung ventilation is widely used for the elimination of tissue hypoxia as well as the method of hyperbaric oxygenation. Artificial blood based on perfluorochemical emulsions as an oxygen-carrying agent has also been developed. It should be noted that all these technologies were developed a long time ago and do not satisfy the requirements of modern medicine that highly needs development of new methods for local tissue oxygenation and elimination.

In our earlier work [5] we proposed a new concept for extracting an additional amount of oxygen from oxyhemoglobin $\left(\mathrm{HbO}_{2}\right)$ by laser-induced photodissociation in cutaneous blood vessels. This new approach is based on studying the interaction of laser radiation with biological tissues, taking into account the absorption of laser light by blood hemoglobin $(\mathrm{Hb})$ and its derivatives $\left(\mathrm{HbO}_{2}\right.$ and $\left.\mathrm{HbCO}\right)$. This enabled the development of an optical technology of increasing the local oxygen concentration in tissue by means of additional extraction of $\mathrm{O}_{2}$ from blood $\mathrm{HbO}_{2}$.

\section{AIM OF THE STUDY}

The aim of this study is an experimental investigation in vivo of the rate of the $\mathrm{HbO}_{2}$ photodissociation in blood vessels under the influence of the transcutaneous laser irradiation in the spectral range from 405 to $940 \mathrm{~nm}$.

\section{OPTICAL METHOD OF TISSUE OXYGENATION}

Absorption of laser radiation by blood hemoglobin $\mathrm{Hb}$ and oxyhemoglobin $\mathrm{HbO}_{2}$ initiates the following primarily physical processes: non-radiative dissipation of electronic excitation energy through heat transfer and photodissociation of $\mathrm{HbO}_{2}$ in blood vessels. In case of low energy laser radiation, a local increase of temperature within $0.1-0.2{ }^{\circ} \mathrm{C}$ may be expected. Such a small rise of a local temperature practically does not lead to any thermal effect. We suppose that in a case of low energy lasers the most important process is the photodissociation of oxyhemoglobin, as a result of which additional molecular oxygen is generated in the tissue (Fig. 1).

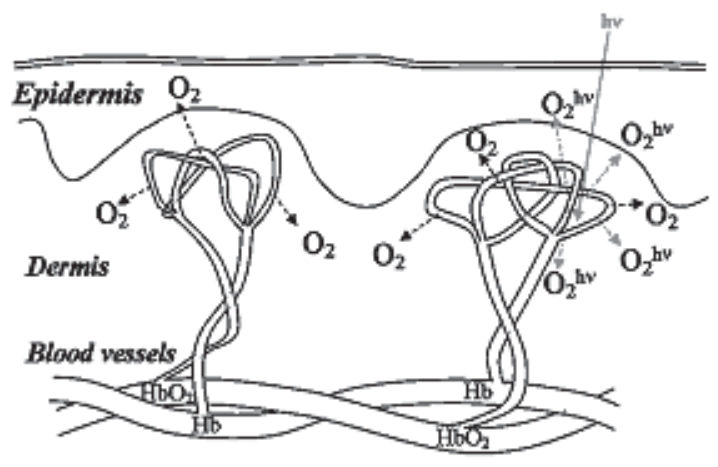

Fig. 1. Illustration of laser-induced tissue oxygenation caused by photodissociation of arterial blood $\mathrm{HbO}_{2}$ 
Quantum efficiency of the photodissociation of $\mathrm{HbO}_{2}$ is sufficiently high and reaches $10 \%$ in a wide visible spectral range. Molecular oxygen is generated due to laser-induced photodissociation of $\mathrm{HbO}_{2}$ in blood vessels, which enables the control of local increase in oxygen concentration in the irradiated region. As a result we obtain an average concentration of oxygen delivered with blood microcirculation and due to laser-induced photodissociation.

$$
\Sigma\left[\mathrm{O}_{2}\right]=\left[\mathrm{O}_{2}\right]+\left[\mathrm{O}_{2}^{\mathrm{hv}}\right]
$$

Investigation of photodissociation of $\mathrm{HbO}_{2}$ in vivo could be carried out using an oxygen saturation parameter. By definition oxygen saturation in pulse oximetry is:

$$
\mathrm{SpO}_{2}=\left\{\left[\mathrm{HbO}_{2}\right] /\left(\left[\mathrm{HbO}_{2}\right]+[\mathrm{Hb}]\right)\right\} 100
$$

Photodissociation of $\mathrm{HbO}_{2}$ induced by laser radiation releases free molecular oxygen. Meanwhile, the proportion between $\mathrm{HbO}_{2}$ and $\mathrm{Hb}$ concentrations is changed and that decreases the value of $\mathrm{SpO}_{2}$.

$$
\Delta \mathrm{SpO}_{2}=\mathrm{SpO}_{2}-\mathrm{SpO}_{2}^{\mathrm{hv}}
$$
radiation.

where $\mathrm{SpO}_{2}$ is the saturation without, and $\mathrm{SpO}_{2}{ }^{\text {hv }}$ the saturation with laser ir-

Direct measurement of this value enables to determine the percentage of oxygen released into the tissue, regardless of the individual optical properties of the skin and radiation parameters.

\section{MATERIAL AND METHODS}

Determination of the relative $\mathrm{HbO}_{2}$ concentration in blood is conducted by a method similar to the method of pulse oximetry which is based on the measurement of light modulated pulse wave of blood. Saturation $\mathrm{SpO}_{2}$ was measured with a pulse oximetry sensor operating in backscattered light based on the standard LED pair with emission wavelengths of 660 and $940 \mathrm{~nm}$ and a photodiode BPW34 (OSRAM). Average initial $\mathrm{SpO}_{2}$ levels (without irradiation) in all groups of measurements differ insignificantly, in the range of 95.0 to $96.0 \%$. Due to the original method of data processing, the accuracy of measurements is better than $0.5 \%$. The system allows continuous photoplethysmographic monitoring, recording and data storing. Data acquisition is executed by a measuring block with a microcontroller connected to a computer.

Measurements were taken on the fingers of healthy non-smoking volunteers with informed consent obtained from each subject and approval by the institutional review board. All procedures performed in the study were in accordance with the ethical standards. The pulse oximetry sensor was placed on the first phalanx of the finger. The light exposure of the finger was carried out by corresponding LEDs (or laser diodes) at 15 wavelengths in the $400-940 \mathrm{~nm}$ spectrum range: 405; 470; 525; $568 ; 590 ; 605 ; 635 ; 660 ; 700 ; 780 ; 860$ and $940 \mathrm{~nm}$. The optical power of every 
source was selected to provide an approximately equal number of incident photons on the irradiated skin area at different wavelengths. The corresponding power density of skin irradiation, while taking into account the output aperture, varied from 50 $\mathrm{mW} / \mathrm{cm}^{2}$ for $\lambda=405 \mathrm{~nm}$ to $125 \mathrm{~mW} / \mathrm{cm}^{2}$ for $\lambda=940 \mathrm{~nm}$.

Two series of experimental measurements were performed. In the first series, the irradiation beam was directed at the lower front of the first phalanx at about $5 \mathrm{~mm}$ distance of the measuring sensor. In the second series, the radiation was directed at the lower part of the second phalanx near the joint between the first and second phalanges. In this case the distance from the irradiation spot to the photodiode was about $15 \mathrm{~mm}$. In both cases direct light flux did not reach the surface of the photodetector. Signals from each light source were recorded as follows: $30 \mathrm{~s}$ with no radiation, $30 \mathrm{~s}$ with radiation and $30 \mathrm{~s}$ without radiation. The mean value of saturation $\mathrm{SpO}_{2}$ on every interval and the change of saturation $\Delta \mathrm{SpO}_{2}$ induced by irradiation were calculated and averaged over the number of records.

\section{RESULTS AND DISCUSSION}

Figure 2 shows a typical example of changing the rate of the $\mathrm{SpO}_{2}$ level in the blood measured with and without LEDs exposure on the first finger phalanx (in this case $635 \mathrm{~nm}$ ). As can be seen, changes in oxygen saturation took place practically immediately when illumination was switched on. During irradiation the $\mathrm{SpO}_{2}$ level remained constant and returned to the initial value immediately after the irradiation shutdown. That can be explained by fast geminate recombination and oxygen rebinding as well as by blood evacuation from the site of measurement due to blood flow. It is evident that the observed local drop of arterial oxygen saturation during irradiation was caused by $\mathrm{HbO}_{2}$ photodissociation in capillary blood vessels.

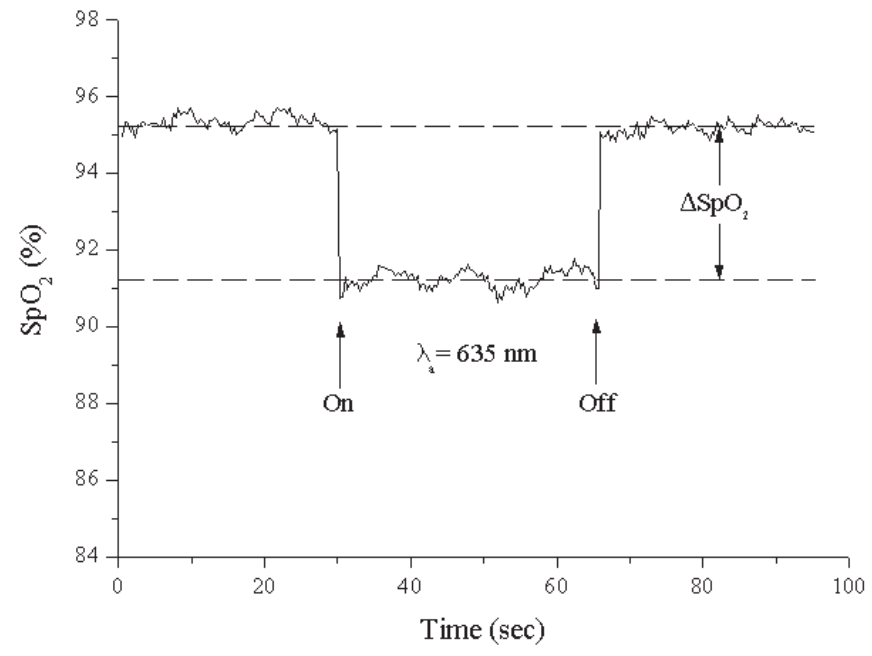

Fig. 2. Changes in the level of $\mathrm{SpO}_{2}$ during irradiation of the first phalanx 
Decrease in the level of oxygen saturation $\Delta \mathrm{SpO}_{2}$ depends on wavelength and power of irradiation. Photoplethysmograms were recorded for all wavelengths in the $400-940 \mathrm{~nm}$ spectrum range. Figure 3 shows the rate of $\Delta \mathrm{SpO}_{2}$ plotted against irradiation wavelength for two series of measurements on the first and the second finger phalanges. In the same figure, for comparison, the $\mathrm{HbO}_{2}$ absorption spectrum (3) is plotted, according to Prahl et al [6].

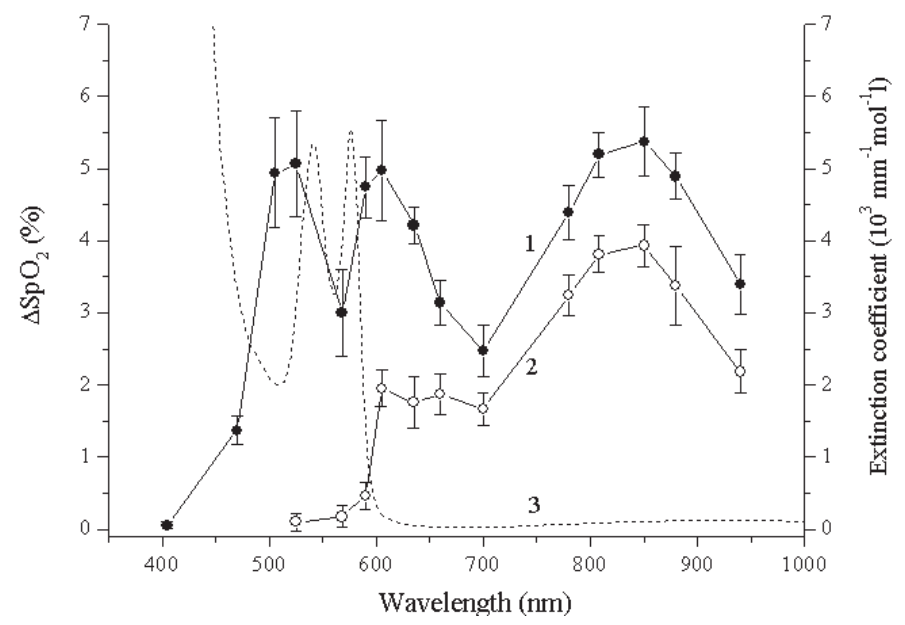

Fig. 3. Change in oxygen saturation $\Delta \mathrm{SpO}_{2}$ during irradiation in the first (1) and the second (2) series depending on the wavelength of the irradiation.

In the first series the oxygen saturation $\mathrm{SpO}_{2}$ dropped up to $5 \%$ from its usual level. Three maxima in the spectral range were revealed, two - in the visible range of spectrum and one broader peak in the near IR range $(850 \mathrm{~nm})$. Two peaks can clearly be seen at near $530 \mathrm{~nm}$ and near $600 \mathrm{~nm}$, which correlated well with calculated (3) absorption spectra. With increasing light path length in tissue which took place in the second series (irradiation of the second finger phalanx) the short-wave peak near $530 \mathrm{~nm}$ disappeared, the peak near $600 \mathrm{~nm}$ decreased considerably and the greatest effect $\left(\Delta \mathrm{SaO}_{2} \approx 4 \%\right)$ was observed near $850 \mathrm{~nm}$. Light with wavelengths below $470 \mathrm{~nm}$ is strongly absorbed in superficial skin layers and irradiation in this spectral range has no effect on oxygen saturation.

The obtained results correlate with the peculiarities of the light propagation in blood filled tissue. Visible light at $450 \mathrm{~nm}$ and $580 \mathrm{~nm}$ has a small depth of penetration into skin tissue, because of their proximity to absorption bands of basic skin chromophores such as oxyhemoglobin and melanin. Therefore, in real tissues the irradiation in this spectral region can cause $\mathrm{HbO}_{2}$ photodissociation only in shallow superficial skin layers. Calculations of effective oxyhemoglobin absorption spectra in the depth of tissue based on the Kubelka-Munk model [7] showed that with increasing light pen- 
etration depth the effective absorption shifted to long-wave spectral region, and from the depth of tissue deeper than $2.5 \mathrm{~mm}$ the IR absorption band of the $\mathrm{HbO}_{2}$ played a dominant role in the absorption of laser radiation. Therefore, it is clear that under light irradiation of more or less considerable tissue volume $\left(\geq 1 \mathrm{~cm}^{3}\right)$ the primary role in $\mathrm{HbO}_{2}$ photodissociation belongs to radiation of red and especially near IR range.

It is interesting and remains unclear what fraction of $\mathrm{O}_{2}$ molecules released by photodissociation can escape from the heme pocket and diffuse through cell membranes and capillary walls thereby increasing tissue oxygen tension. The data obtained in the present study demonstrated the saturation drop only during irradiation, but did not give any information about possible change in free oxygen content in tissue.

On the other hand, Asimov et al. [8] measured a significant increase in tissue oxygen tension $\mathrm{pO}_{2}$ during irradiation with $\mathrm{He}-\mathrm{Ne}$ laser $(633 \mathrm{~nm}, 1 \mathrm{~mW})$. Measurements were carried out using transcutaneous membrane sensor with Clark electrode on the internal side of forearm of three volunteers. A Clark electrode was placed close to irradiation spot with diameter of $2.5 \mathrm{~mm}$. After 10 minutes of irradiation the local oxygen tension increased up to 1.6 times in all three patients (Fig. 4). In the case of artificially induced ischemia additional extraction of oxygen was also observed. The obtained data demonstrated the partial release of oxygen into tissue during $\mathrm{HbO}_{2}$ photodissociation.

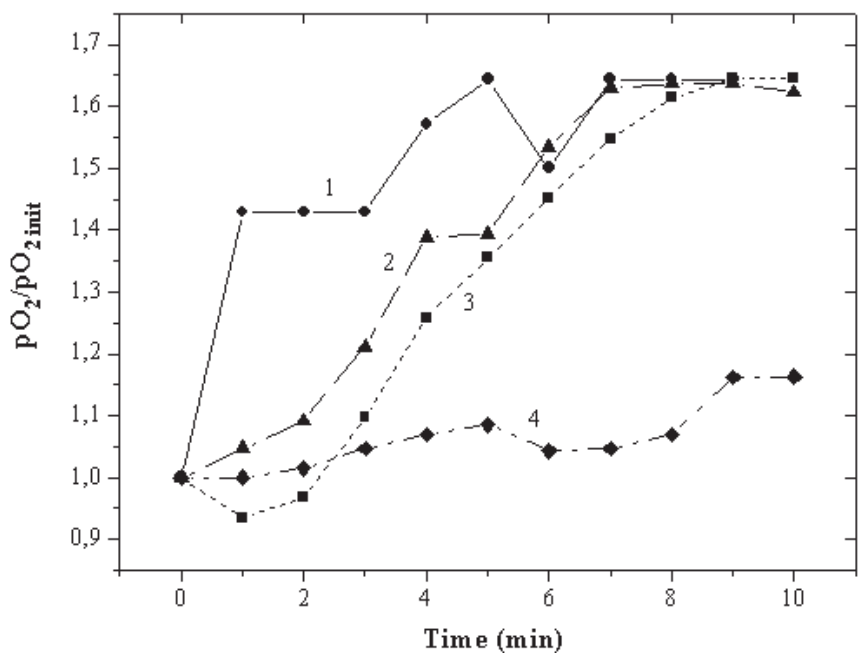

Fig. 4. Relative changes in tissue oxygen tension in three patients with normal microcirculation (1-3) and artificial ischemia (4) during irradiation with He-Ne laser.

Thus, we can expect that after enough prolonged irradiation (several minutes), a certain fraction of oxygen molecules released due to photodissociation of $\mathrm{HbO}_{2}$ will diffuse in surrounding tissue and increase the oxygen partial pressure. A possibility to increase the free oxygen content in tissues can be applied in clinical practice for 
treatment of a series of diseases related to violations of the microcirculation and oxygen supply and therefore requires further investigations.

\section{CONCLUSION}

A new optical method for reduction of local tissue hypoxia is proposed. The value of tissue oxygen concentration increases significantly during the laser irradiation. The rates of oxygen saturation $\mathrm{SpO}_{2}$ in blood in vivo and their dependence on the wavelength of the transcutaneous laser irradiation have been experimentally measured. The observed reduction in $\mathrm{SpO}_{2}$ up to $5 \%$ indicates the process of photodissociation of $\mathrm{HbO}_{2}$ in vivo and may result in the local $\mathrm{O}_{2}$ growth in the tissue. The obtained results correlate with the peculiarities of the light propagation in blood filled tissue. Near IR radiation plays a dominant role in absorption of laser radiation by oxyhemoglobin in deeper layers of tissue blood vessels. The obtained results can be used to improve the efficiency of laser therapy.

\section{Acknowlegement}

This work is partially supported by the project DFNI B02/9 /2014 of the Bulgarian National Science Fund.

\section{REFERENCES}

1. Rodriguez, P., Felix, F. The role of oxygen in wound healing: a review of the literature. - Dermatol. Surg., 34, 2008, 1159-1169.

2. Baxter, G. D. Therapeutic lasers: Theory and Practice. Edinburgh, New-York, 1994

3. Fuchs, J. The role of oxygen in cutaneous photodynamic therapy. - Free Radic. Biol. Med. 15, 1998, 835-847.

4. Vaupel, P. Oxygenation of Human Tumors. - Strahlenther Onkology. 166, 1990, 377-386.

5. Asimov, M., Asimov, R., Mirshahi, M., Gisbrecht, A. Effect of laser induced photodissociation of oxyhemoglobin on biomedical processes. - Proc. SPIE., 4397, 2001, 390-394.

6. Prahl, S. Optical Absorption of Hemoglobin. - Tech. Rep. Oregon Medical Laser Center Portland Oregon USA., 1999

7. Asimov, M., et al. Investigation of the efficiency of laser action on hemoglobin and oxyhemoglobin in the skin blood vessels. - Proc. SPIE.,3254, 1998,407-412.

8. Asimov, M., Thanh, N. Laser-induced photodissociation of oxyhemoglobin: Optical method of elimination of hypoxia. - Optics and Spectroscopy., 111, 2011, 254-259.

\section{Corresponding author:}

Alexander Gisbrecht

Institute of Electronics

Bulgarian Academy of Sciences

72 Tzarigradsko Shausse Blvd.

1784 Sofia, Bulgaria

( 0887834893

e-mail: aigiz@abv.bg 